\title{
PSICOEDUCAÇÃO FAMILIAR
}

\author{
FAMILY PSYCHOEDUCATION \\ PSICOEDUCACIÓN FAMILIAR
}

J uliana Yacubian*

Francisco Lotufo Neto**

\begin{abstract}
RESUMO: Os avanços obtidos pela psiquiatria no tratamento das pessoas com transtorno mental permitiram nos últimos 50 anos que o número de internações e sua duração diminuíssem, permitindo a vida próxima à comunidade. Com isto o papel da família no cuidado do doente mental tornou-se mais importante. A família foi considerada no passado recente, causadora ou mediadora da doença mental. Isto gerou grandes problemas para o tratamento eficiente do paciente. Mais recentemente, o papel como responsável pelo cuidado com o doente mental vem sendo valorizado. Para essa atividade, a educação básica sobre doença mentalé imprescindível. A psicoeducação familiar traz benefícios para os pacientes (diminui recaídas) e familiares (diminui sobrecarga). As reuniões psicoeducacionais aqui descritas fornecem informações científicas às famílias e permitem troca de experiências sob a forma de aulas e reuniões em pequenos grupos.
\end{abstract}

DESCRITORES: Saúde da família; Saúde mental; Psicologia educacional; Enfermagem psiquiátrica; Família.

\section{INTRODUÇÃO}

Com a evolução dos tratamentos psiquiátricos, os indivíduos portadores de transtornos mentais vêm sendo mantidos na comunidade e acompanhados ambulatorialmente pelos serviços de saúde mental. Desse modo, a responsabilidade pelos cuidados ao doente mental passa a ser menos das instituições e mais das famílias, os familiares tornam-se responsáveis pelos cuidados primários (Hatfield, 1987).

As famílias proporcionam ao paciente o suprimento de suas necessidades físicas, suporte financeiro, os mantém a salvo do ambiente, ensinam novas habilidades, organizam atividades sociais e recreacionais, monitoram a medicação e consultas médicas e socorrem os pacientes repetidas vezes nas situações de crise. A família passa a ser a interface entre o doente mental e o sistema de saúde mental (Domenici \& GriffinFrancell, 1993).

F reqüentemente, porém, não é fornecido aos familiares ou a comunidade em geral informações básicas ou um treinamento formal adequado para o manejo diário destes indivíduos, muitas vezes tornando-os uma sobrecarga para a família e com isso podendo causar prejuízos para o doente mental e para a sociedade (Winefield \& Harvey, 1994). Os familiares geralmente sentem-se despreparados para ajudar um parente com doença mental e apresentam muitas dúvidas. 0 convívio com o paciente portador de transtorno psiquiátrico pode produzir uma grande sobrecarga que acaba por comprometer a saúde, vida social, relação com os outros membros da família, lazer, disponibilidade financeira, rotina doméstica, desempenho profissional e escolar e inúmeros outros aspectos da vida dos familiares. Os cuidadores que dedicam-se aos pacientes mais debilitados investem tempo e energia na busca de tratamento e nas negociações para que eles aceitem se tratar. A interação com os serviços de saúde mental também é uma fonte de sobrecarga, pois na maioria das vezes os contatos são vivenciados como uma experiência frustrante, confusa e humilhante (Hatfield, 1978).

Apesar dos intensos transtornos e do sofrimento observado, as famílias dos doentes mentais quase sempre aceitam conviver com os pacientes e suportam toda a sobrecarga que lhes é imposta. Independentemente

Recebido em 05/06/01 aceito em 10/10/01

\footnotetext{
* Pós-graduanda e Supervisora da Residência em Psiquiatria do Instituto de Psiquiatria do Hospital das Clínicas da Faculdade de Medicina da Universidade de São Paulo.

** Professor Associado do Departamento de Psiquiatria da Faculdade de Medicina da Universidade de São Paulo.
} 
da ideologia dos serviços de saúde, da renda "per capita" do país, do tempo e número de internações, enfim, de inúmeras outras variáveis, as famílias ainda representam a principal alternativa ao hospital psiquiátrico (Bachrach $\&$ Clark, 1996).

Apesar dos avanços da psiquiatria, o número de serviços de saúde mental que oferecem programas específicos de apoio para os cuidadores e que os aceitam como aliados na elaboração dos projetos terapêuticos e de reabilitação é extremamente reduzido. Infelizmente, na maioria dos serviços o único papel reservado para a família é o de agente custodial e, normalmente, não considera-se a necessidade que eles têm de receber informações sobre a doença, de expressarem os seus pontos de vista e as suas dificuldades.

Para auxiliar estas famílias à educação sobre saúde mental, que chamaremos de psicoeducação, vem sendo implantada em algumas instituições que cuidam de saúde mental e sua importância mostra-se cada vez mais evidente (Anderson et al.,1980; McFarlane et al., 1993.). A função da psicoeducação é de ensinar aos membros de uma família que cuidam de um doente mental sobre a doença em si, os tratamentos, as necessidades de um portador de transtorno mental quanto suas capacidades de desenvolvimento e habilidades, prevenção de recorrências e convivência harmônica.

\section{INTERAÇÃO FAMILIAR E DOENÇA MENTAL}

As relações entre família e doença mental vêm sendo estudadas há algum tempo, principalmente na Esquizofrenia e só mais recentemente em outras patologias psiquiátricas, como o Transtorno Bipolare Depressão.

Kasanin et al (1934), em um primeiro ensaio sistemático sobre a relação entre família e esquizofrenia, apontaram um alto índice de superproteção e rejeição em mães de pacientes esquizofrênicos. S egundo estes autores, estas mães eram capazes de detectar a inferioridade biológica da criança que iria desenvolver esquizofrenia e por isto superprotegiam estas crianças.

As relações entre interação familiar e doença mental tem evoluído nos últimos quarenta anos. Três principais hipóteses mantém entre si um vínculo evolutivo e conceitual (Halford, 1992). Estas hipóteses são: 1) distúrbios na interação familiar são a causa da doença mental; 2) certos tipos de interação familiar são mediadoras do curso da doença mental estabelecida; 3) as famílias são um importante recurso na reabilitação de doentes mentais e, embora estejam submetidas à sobrecarga que a luta contra a doença mental pode ocasionar, fazem a ligação entre o doente mental e os serviços de saúde, sendo aliadas no tratamento.

\section{EVOLUÇÃO HISTÓRICA - FAMÍLIA COMO CAUSA DA DOENÇA MENTAL}

Historicamente, a primeira hipótese sobre as relações entre família e esquizofrenia era a de que estilos de relações familiares disfuncionais causavam esquizofrenia (Fromm-R eichmann, 1948; Laing, 1965; Wynne \& Singer, 1963). Seguindo teorias psicanalíticas Fromm Reichmann em 1948, criou o famoso termo "mãe esquizofrenogênica" no qual estava implícita a responsabilidade da relação mãe e filho no, desenvolvimento da psicose e conseqüentemente da esquizofrenia. Essa autora, entretanto, defendia o envolvimento das mães no tratamento e reabilitação de pacientes psicóticos. Em um de seus livros (P rinciples of Intensive Psichotherapy - Fromm Reichmann, 1948), descreve a importância de ensinar as mães de pacientes psicóticos a mudarem seus comportamentos em relação a os filhos e o quanto isso podia auxiliar no curso favorável destes pacientes.

Batenson e a escola de Palo Alto, em 1956, desenvolvem a teoria de duplo-vínculo, na qual ocorreria uma relação entre duas ou mais pessoas onde fossem constantemente emitidas mensagens contraditórias e mutuamente excludentes, deixando o receptor das mensagens sem saída diante do emissor. Segundo estes autores esse tipo de comunicação desde a infância poderia levar ao desenvolvimento de uma fragilidade psíquica com o desencadeamento de doenças mentais (Batenson et al., 1956).

Lidz e seu grupo, em 1957, lançam a idéia de "cisma conjugal" na qual a identificação das crianças com um dos cônjuges é atacada e hostilizada pelo outro criando uma divisão familiar. Este tipo de acontecimento 
estaria presente em famílias de crianças que iriam tornar-se esquizofrênicos desde os primeiros meses de vida (Lidz et al.1957).

Wynne et al.(1963), através de um estudo comparando famílias de pacientes esquizofrênicos com famílias de pacientes psiquiátricos não esquizofrênicos demonstrou alterações de comunicação e organização intrafamiliar presentes precocemente na composição das famílias de pacientes esquizofrênicos.

A té aonde vão os conhecimentos da psiquiatria? Atualmente está claro que interações familiares disfuncionais isoladamente não causam transtornos psiquiátricos.

\section{FAMÍLIA COMO MEDIADORA DO CURSO DA DOENÇA MENTAL}

Estudos britânicos, comandados por B rown, et al.(1958), sugeriam que pacientes psiquiátricos convivendo com uma família fechada tinham índice maior de recaídas quando comparados a pacientes psiquiátricos que residiam em albergues, distantes de seus parentes. Em uma replicação deste estudo foi encontrado uma evolução favorável em pacientes morando junto com irmãos quando comparados com pacientes que moravam com os pais ou esposas, sendo que nestes estudos cuidou-se para que essas diferenças não pudessem ser explicadas pela gravidade ou duração da doença, sugerindo que algumas características do ambiente familiar poderiam predispor aos índices de recaídas dos pacientes.

A partir destes achados Brown, et al. (1962), hipotetizaram que pacientes vivendo próximos de seus parentes poderiam estar expostos a altos níveis de afetos negativos ou intrusão, aumentando a vulnerabilidade a recaídas. Ao longo de vinte anos de estudos sistemáticos, este grupo de pesquisadores formulou que o tipo de ambiente em que os pacientes esquizofrênicos vivessem após a alta hospitalar influenciaria grandemente no seu prognóstico a curto e longo prazo.

O Camberwell Family Interview (CFI), desenvolvido por Brown \& Rutter (1966), é um instrumento de entrevista semi-estruturado usado para determinar as condições afetivas da família que convive com um paciente psiquiátrico. Este instrumento foi criado para a obtenção de informações sobre o ambiente familiar e expressões emocionais. É aplicado em familiares quando um paciente esquizofrênico mostra piora dos sintomas ou é admitido em um hospital para tratamento. Esta entrevista é feita individualmente com cada familiar e o paciente não está presente durante a entrevista. $O$ termo Emoção Expressa $(E E)$, foi criado para designar o estresse causado por um ambiente familiar "negativo". EE se refere a atitudes direcionadas ao paciente, como expressão da crítica, hostilidade, ou superenvolvimento emocional (auto-sacrifícios extremos). Os parentes são classificados como tendo alta EE na CFI se eles fazem críticas freqüentes ao paciente, expressam hostilidade ou atitudes de rejeição, ou exibem sinais de pronunciado superenvolvimento emocional.

Pesquisas retrospectivas têm mostrado forte evidência de que EE familiar é preditora de recaídas de sintomas dentro do período que se segue a alta hospitalar do paciente que estava sendo tratado de uma exacerbação aguda.

A relação entre EE e recaída tem sido extensivamente estudada para pacientes com esquizofrenia, com mais de vinte e cinco estudos conduzidos com mais de mil pacientes (Kavanagh, 1992). Outros estudos tem mostrado a utilidade da EE em predizer recaídas em pacientes com Depressão e Transtorno Bipolar, sendo que a EE como instrumento operacional já foi validada para Transtorno Bipolar (Miklowitz, 1988). Os resultados de pesquisa de EE estão ilustrados no Gráfico 1. 
GRÁFICO 1- VULNERABILIDADE A RECAÍDA EM 9 MESES APÓS O RETORNO DE PACIENTES A CONVIVÊNCIA COM FAMILIARES DE ALTA EMOÇÃO EXPRESSA OU FAMILIARES DE BAIXA EMOÇÃO EXPRESSA (adaptado de Mauser \& Glynn, 1995)

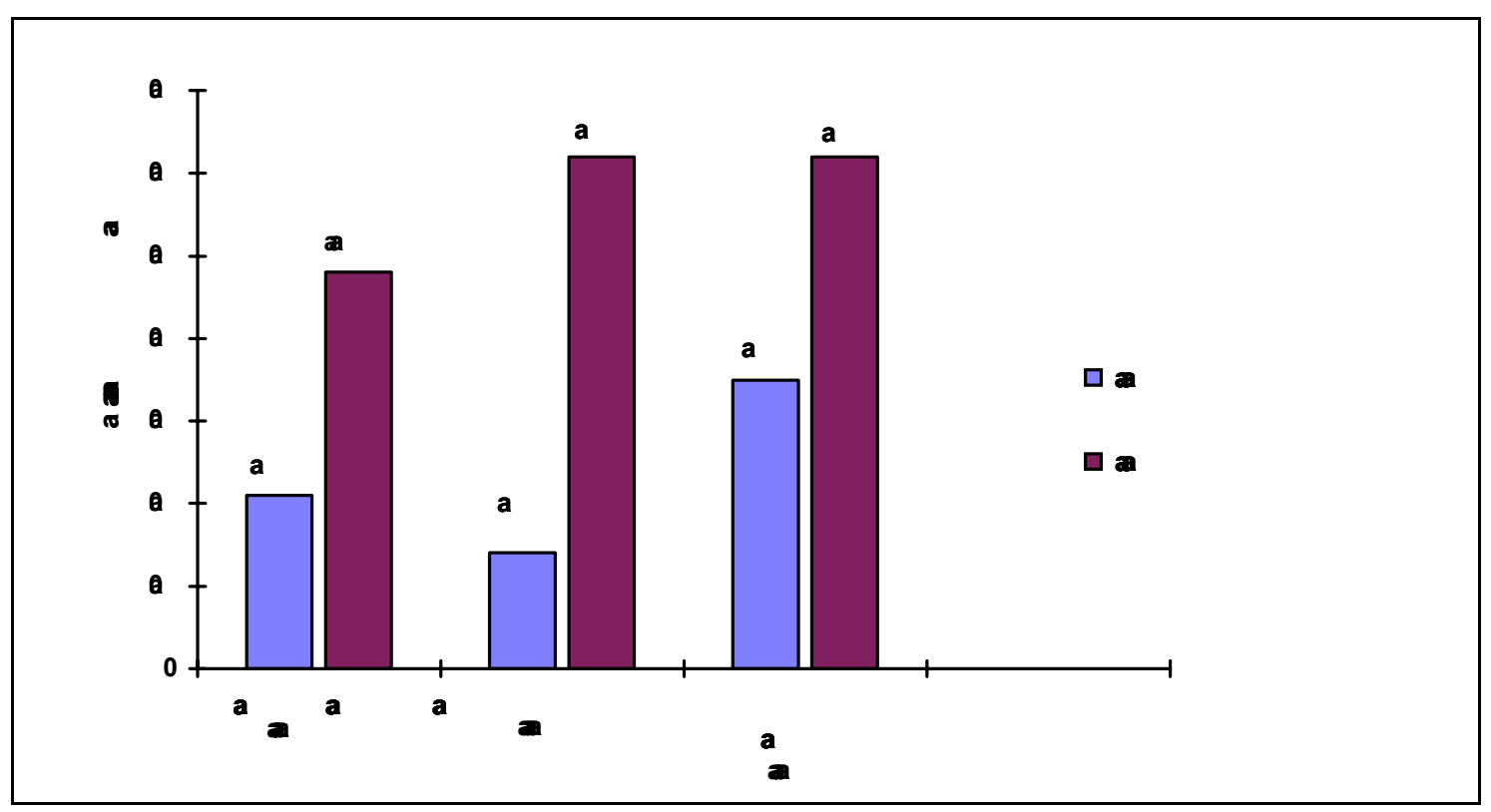

Pode-se ver pelo gráfico que pacientes esquizofrênicos ou bipolares vivendo com parentes de alta EE tem duas vezes mais chance de recair durante os nove meses após a alta hospitalar quando comparados com pacientes que convivem em família com baixa $\mathrm{EE}$. A vulnerabilidade a recaídas também é maior para pacientes deprimidos que convivem com parentes com alta EE.

Estudos sobre EE e padrões de comunicação familiar influenciaram a valorização de intervenções familiares em doenças psiquiátricas. Sendo que, comportamentos negativos de familiares, tais como, críticas freqüentes, hostilidade e intrusão são melhores vistas como uma resposta normal a intensa sobrecarga de se conviver com a doença mental mais do que sintomas de uma psicopatologia familiar.

A presença de interações familiares carregadas negativamente sublinha a importância de se estabelecer uma relação colaborativa entre profissionais e familiares para melhorar a luta contra as doenças psiquiátricas (Mintz, et al. 1987; Kuipers \& Bebbington, 1990).

\section{FAMÍLIA COMO PROVEDORA DE CUIDADOS COM DOENTE MENTAL}

Até agora vimos o impacto da interação familiar sobre o portador de doença mental. Mas, como considerar o impacto que a convivência e os cuidados com um doente mental podem trazer para uma família? $\mathrm{Na}$ língua inglesa utiliza-se a palavra "burden". Na língua portuguesa a palavra que mais se aproxima deste conceito é sobrecarga.

Os efeitos negativos de doenças psiquiátricas graves no funcionamento familiar vêm sendo amplamente documentados em estudos que tentam padronizar questionários e medidas para aferir o nível de sobrecarga que um doente mental pode trazer para uma família e quais estratégias as famílias acabam desenvolvendo para lidar com esta sobrecarga (Hatfield, 1987; J ackson, et al., 1990; Mueser et al., 1997).

Os membros da família de um doente psiquiátrico freqüentemente têm que aprender como lutar com uma série de problemas: diminuição de cuidados pessoais, agressividade, comportamentos inapropriados, falta de adesão ao tratamento, pronunciado isolamento social, risco de suicídio, mudanças de humor, ansiedade e depressão pervasivas, abuso de substâncias, comportamentos maníacos, comportamentos delirantes e alucinatórios. A falta de previsibilidade que ocorre em uma relação com um doente psiquiátrico tem efeito negativo profundo entre os membros da família. 0 comportamento errático de muitos pacientes psiquiátricos, associado a falta de informações acuradas sobre a doença mental freqüentemente são causas de estresse e tornam os membros da família suscetíveis a ansiedade, depressão e raiva (Mueser \& Glynn, 1995). 
0 estresse vivenciado por parentes que convivem com um membro da família mentalmente enfermo pode ser documentado em um estudo conduzido por S paniol (1987), que entrevistou 140 membros da National Alliance for the Mentally III (organização americana). A tabela 1 resume as porcentagens de sintomas que os familiares de pacientes com doença mental relataram.

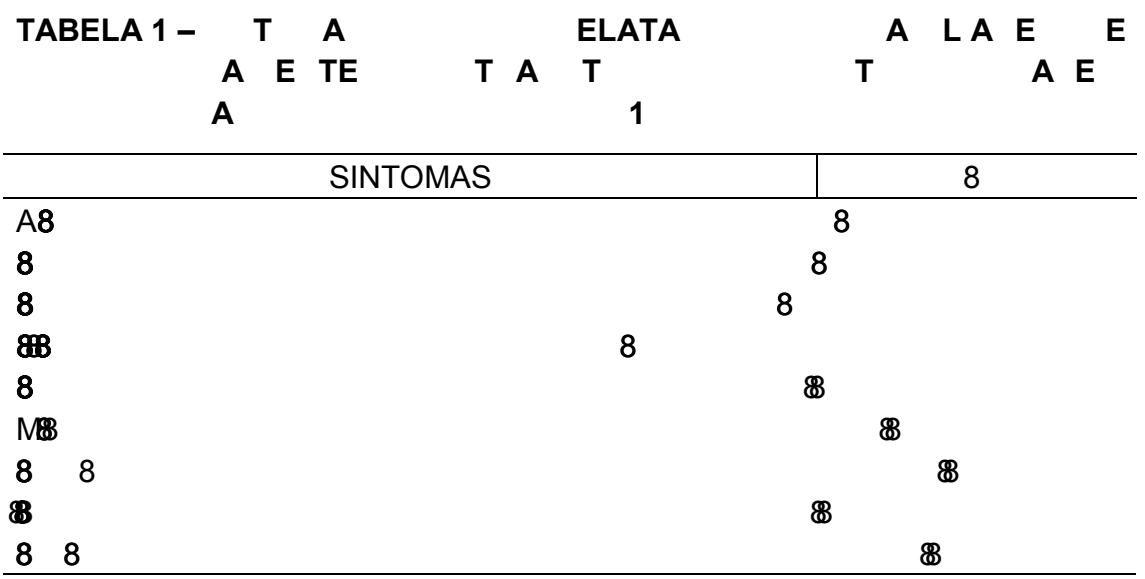

A valorização das queixas e problemas familiares acarretados pela convivência com um doente mental vem ocorrendo desde a década de setenta (Kreisman \& J oy, 1974). Assim como, fornecimento de educação básica sobre doenças psiquiátricas vem se desenvolvendo a partir desta época. Estão sendo desenvolvidas medidas de sobrecarga familiar ocasionada pela doença mental (Levene et al., 1996) e estudos também são realizados no sentido de se criar meios de alívio para esta sobrecarga.

As famílias estão passando a adquirir uma nova condição em relação aos transtornos psiquiátricos, suas necessidades estão sendo estudadas e sua participação no tratamento passa a ser mais ativa, como parte integrante da equipe de cuidados com o doente mental. Os membros das famílias de doentes mentais passam a ser considerados como o alicerce do tratamento, quem com maior precisão pode monitorar as manifestações da doença e a eficácia da terapêutica. Mas, sem informações básicas sobre os sintomas, curso e tratamento da doença as famílias não podem tornar-se membros efetivos da equipe de cuidados com 0 doente mental, dependendo sempre de monitoração profissional com aumento de custo e sobrecarga tanto para as famílias quanto para os serviços de saúde mental.

\section{EVIDÊNCIAS DE EFICÁCIA}

Como inicialmente os métodos psicoeducacionais eram aplicados apenas às famílias de pacientes esquizofrênicos os estudos sobre eficácia destes métodos por este período inicial limitaram-se a este grupo de pacientes. Mais recentemente, principalmente a partir de década de noventa, quando os métodos psicoeducacionais começaram a ser aplicados para outras patologias psiquiátricas, como Transtorno Afetivo Bipolar e Depressão, foi que se iniciaram também as tentativas de se medir a eficácia de métodos educacionais para estas patologias.

As tentativas para se avaliar a eficácia da psicoeducação familiar tem sido testadas amplamente por vários grupos que estudam famílias de portadores de esquizofrenia, existem algumas evidência de que ocorra uma redução nos níveis de recaída de esquizofrênicos cujas famílias participam de intervenção psicoeducacional quando comparados a pacientes que seguem apenas o tratamento ambulatorial, is to é, tem apenas a conduta medicamentosa sem a intervenção educacional familiar (Beardslee et al., 1997; Penn \& Mueser, 1996; Macfarlane et al., 1996).

Em uma ampla revisão de estudos controlados sobre a eficácia de tratamentos psicossociais em esquizofrenia, realizada por Penn \& Mueser (1996), foi evidenciado que intervenção familiar de orientação educacional reduz a vulnerabilidade a recaídas dentro de um a dois anos de seguimento. Ainda nesta revisão foram encontrados dois estudos mostrando uma redução dos níveis de sobrecarga familiar após intervenções familiares educacionais. Estes autores concluem, baseados nesta revisão, que intervenções familiares de orientação educacional proporcionam benefícios para ambos pacientes e familiares. 
Os vários métodos psicoeducacionais que fornecem informação, suporte, treinamento de habilidades ou uma combinação destes, mostram-se efetivos em atingir as necessidades das famílias. Além disso as famílias que recebem esse tipo de educação tornam-se capacitadas para exercerem algum controle sobre as intervenções médicas e não apenas sentirem-se culpadas por terem familiares doentes e se colocarem em posição passiva como se fossem unidades patogênicas (Mueser \& Glynn, 1995).

Zipple \& S paniol (1987), sugerem que a eficácia dos diversos métodos psicoeducacionais são similares pois os níveis de recidiva são muito amplos para que possam capturar diferenças significativas entre os métodos, então, outras medidas são recomendadas por estes autores para se verificar a qualidade dos métodos psicoeducacionais fornecidos as famílias, como: satisfação familiar com o método empregado, nível de funcionamento do paciente, nível de funcionamento familiar, nível de estresse familiar.

A maioria das pesquisas realizadas em psicoeducação envolvem famílias que vivem com seus parentes doentes e que são avaliadas como tendo um alto grau de EE (Hogarty et al., 1986).

Medidas de psicoeducação familiar mostram-se úteis para diminuir a $E E$ e conseqüentemente as recaídas da doença. Estas medidas ensinam os pacientes e suas famílias a lidarem com a doença psiquiátrica de maneira a ter um entendimento pragmático da doença, dos sintomas, das estratégias de manejo de comportamentos e de onde e quando procurar auxílio.

Os trabalhos que testam a eficácia da psicoeducação familiar em Transtornos Afetivos são bem mais raros. Keitner (1990), diz que apesar de ainda não existirem estudos controlados sobre a eficácia de psicoeducação familiar em Depressão, os membros das famílias e pacientes têm fornecido retorno intensamente positivo quando este tipo de abordagem é fornecida. Beardslee et al. (1997), desenvolveram um estudo piloto com o objetivo preventivo de doença afetiva em filhos de pais com o diagnóstico de depressão, demonstraram benefícios da psicoeducação para famílias onde, o pai e/ou a mãe apresentavam um episódio depressivo recente ao início da intervenção, neste estudo os resultados foram examinados após um ano e um ano e meio de intervenção psicoeducacional, mostrando uma manutenção dos benefícios da psicoeducação a longo prazo.

As medidas da eficácia da psicoeducação vem ganhando importância a medida que este tipo de prática vem sendo instalada em vários serviços de saúde mental.

\section{METAS DA PSICOEDUCAÇÃO FAMILIAR}

S egundo Anderson et al. (1986), as metas da educação familiar não se limitam a um conhecimento da doença psiquiátrica que traga benefícios para o paciente apenas, mas que atinjam as expectativas familiares no sentido de implementar seus recursos na luta contra a doença e favorecer uma melhor interação familiar.

Q uatro metas principais da educação podem ser identificadas, estas metas estão resumidas no Quadro 2 e a base para cada uma das metas são discutidas a seguir.

QUADRO 1 - METAS DA EDUCAÇÃO FAMILIAR (Modificada de Mueser \& Glynn, 1995).

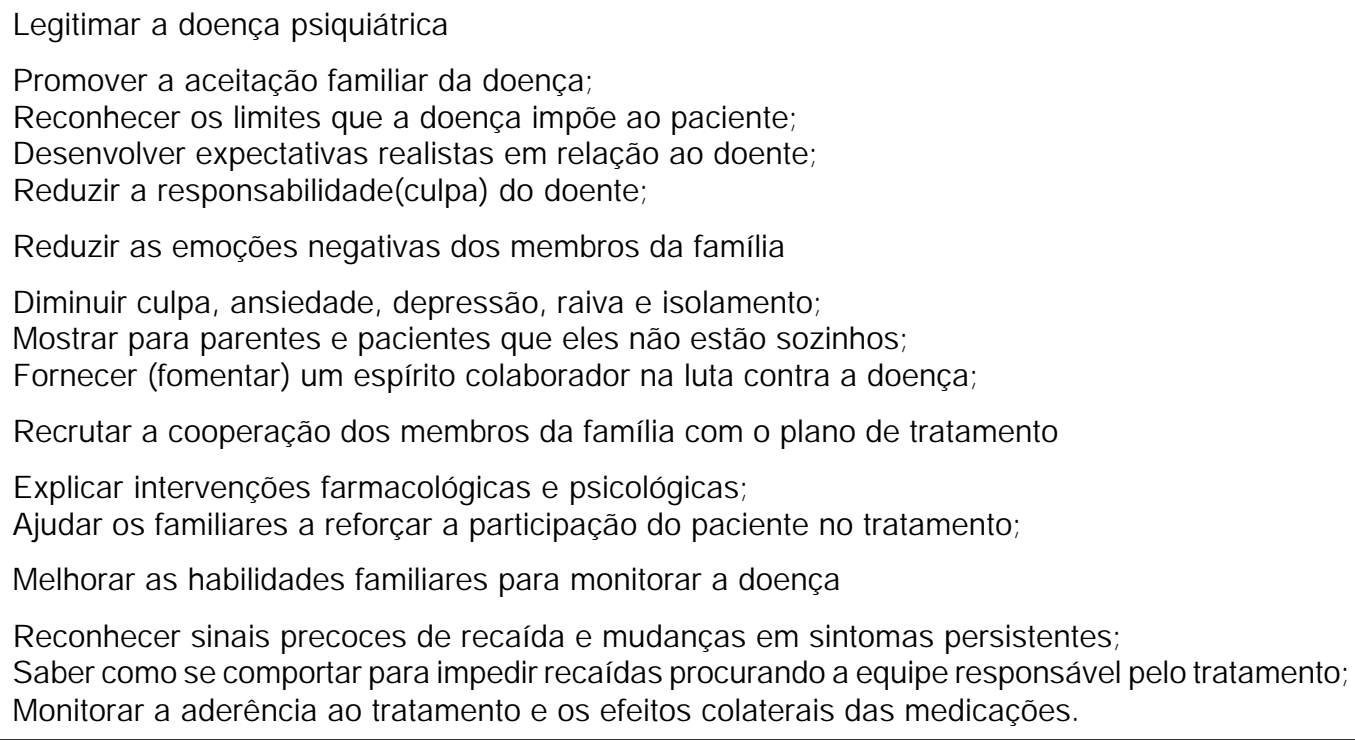




\section{Legitimar a doença psiquiátrica}

A maioria dos sintomas de doenças não psiquiátricas (por exemplo, tosse, febre, hipertensão) são facilmente reconhecidos como conseqüências de problemas fisiológicos que permanecem fora do controle do paciente. Em comparação, os sintomas psiquiátricos (isto é, como ansiedade, depressão, isolamento social, entre outros) são menos aceitos como reflexo de uma doença e mais freqüentemente se acredita que os pacientes tenham um controle voluntário sobre estas manifestações.

A meta para a legitimação da doença psiquiátrica é alcançada através do fornecimento de informações sobre os sintomas da doença e como seu diagnóstico é estabelecido. Ajudando os membros da família a entender que os problemas dos pacientes estão submetidos a um diagnóstico psiquiátrico específico (com uma base biológica real), os familiares tornam-se capazes de entender melhor a doença e os limites que ela pode impor aos pacientes. Através do reconhecimento destes limites que a doença impõe aos pacientes, os familiares desenvolvem expectativas mais realistas em relação ao paciente, ao mesmo tempo que reduz a deposição de culpa sobre o paciente em relação a falta de controle sobre alguns comportamento. Ainda a legitimação da doença reforça a responsabilidade do doente em algumas áreas do seu comportamento.

Reduzir as emoções negativas dos membros da família

Como discutido anteriormente, os parentes de pacientes psiquiátricos estão submetidos a uma enorme gama de emoções negativas, incluindo culpa, depressão, ansiedade, raiva, entre outras. Embora algumas destas emoções sejam inevitáveis, a educação familiar sobre transtornos psiquiátricos pode aliviar alguns destes sentimentos desagradáveis que muitas vezes estão relacionados a um conhecimento precário sobre a doença. Sentimentos negativos podem ser gerados pela luta muitas vezes frustrante contra a doença psiquiátrica. As famílias freqüentemente se sentem sozinhas com suas experiências. A través da informação, a família toma conhecimento que existem outras pessoas e famílias que sofrem com a mesma doença e percebem que eles não estão sozinhos ao entrar em contato com outras famílias e profissionais de saúde mental.

A diminuição das emoções negativas dos familiares também leva a uma diminuição da EE familiar com benefícios para a família e para o paciente.

\section{Recrutar a cooperação dos membros da família com o plano de tratamento}

A recuperação da maioria das doenças psiquiátricas graves necessita de intervenções farmacológicas e psicossociais. Estas intervenções necessitam da colaboração do paciente e o suporte familiar é vital para 0 início e prosseguimento da terapêutica. Para um melhor aproveitamento das intervenções terapêuticas é necessário que a família e o paciente estejam seguros sobre os benefícios e efeitos colaterais dos tratamentos, saibam porque aqueles tratamentos foram escolhidos e possam participar de maneira ativa na escolha de melhores meios de tratamento do seu familiar.

\section{Melhorar as habilidades familiares para monitorar a doença}

Considerando a natureza episódica da maioria dos transtornos psiquiátricos, a monitoração cuidadosa e uma rápida intervenção contra sintomas precoces de exacerbação da doença são fundamentais para prevenção de recaídas e re-hospitalizações. As famílias mantém, freqüentemente, um contato mais próximo com os pacientes do que os profissionais de saúde mental. Por esta razão, uma das metas da psicoeducação é a de tornar os familiares aptos para monitorar o curso da doença e capazes de alertar os profissionais para possíveis recaídas. Com a educação sobre o tratamento farmacoterápico, os familiares podem ainda, colaborar na monitoração da adesão ao tratamento e controle de efeitos colaterais das medicações de maneira mais acurada. Esta monitoração mais consciente do tratamento e dos sintomas da doença poderá ter importantes implicações na evolução da doença a longo prazo. 


\section{MODELOS DE TRABALHO}

A informação aos pacientes e famílias sobre as doenças psiquiátricas pode ser fornecida de várias maneiras, incluindo, transmissão do conhecimento de modo escrito por livros, folhetos informativos, apostilas sobre uma doença específica ou através da transmissão oral do conhecimento no decorrer do tratamento do paciente. A transmissão oral do conhecimento pode ser feita para uma única família isoladamente ou para várias famílias em sessões em grupo. Estes grupos de família (Multiple-Family Groups) originalmente eram reunidos para tratamento psicoterápico sem estar associados a psicoeducação. A partir de necessidades destes grupos passou-se a associar sessões de psicoeducação com sessões de psicoterapia (McFarlene et al., 1995; Anderson etal., 1980). Segundo Anderson et al. (1986), o trabalho em grupo é vantajoso em relação ao trabalho individual das famílias uma vez que, um grupo parece menos ameaçador àqueles membros da família que podem ser relutantes para expor suas dúvidas se estiverem apenas com sua família. 0 grupo pode diminuir a sensação de isolamento familiar e os familiares podem comparar experiências e encontrar conforto; aprendendo que não estão sozinhos nos seus esforços. Há ainda o favorecimento de uma relação menos formal entre as famílias e os profissionais.

A criação dos workshops psicoeducacionais por Anderson, et al. (1980), partiu da necessidade de educação das famílias de pacientes com esquizofrenia com as quais trabalhavam sem a obrigatoriedade destas famílias estarem ligadas a um grupo psicoterápico.

0 termo workshop na língua inglesa originalmente significa oficina, mas em uso mais recente do termo vemos o significado de "curso intensivo sobre um tema específico". 0 termo workshop continuará sendo usado para facilitar a comunicação, uma vez que já é empregado usualmente no meio médico.

O workshop psicoeducacional é um modelo de educação sobre doenças mentais para pacientes e familiares gerado e desenvolvido para a Esquizofrenia por Anderson, et al. (1980), (S urvival Skills Workshop). Posteriormente este modelo foi transportado para Transtornos Afetivos (Clarkin et al., 1992).

\section{OS WORKSHOPS PSICOEDUCACIONAIS}

Os workshops são reuniões com familiares de pacientes que possuem o mesmo diagnóstico. Ocorrem periodicamente durante um dia inteiro.

Esses workshops sobre diagnósticos-específicos tem os seguintes objetivos:

1) proporcionar importantes informações científicas com um custo menor do que trabalhar individualmente os familiares;

2) servem como uma oportunidade para familiares trocarem suas experiências com profissionais e outras famílias;

3) estimula famílias hesitantes a participarem com mais entusiasmo da escolha de melhores meios de cuidado do doente mental;

4) os profissionais tem oportunidade de aprender com as experiências dos membros das famílias a lidar com seus pacientes.

S egundo Anderson et al. (1986), as informações aos participantes dos workshops psicoeducacionais devem seguir alguns princípios: a) Os psicoeducadores devem ter um conhecimento sólido sobre as doenças psiquiátricas; b) As informações devem ser fornecidas de maneira honesta e direta, isto é, não se deve negar informações para "proteger" a família e deve-se fornecer a melhor informação possível, reconhecendo os limites do conhecimento profissional quando apropriado; c) Deve ser um processo interativo entre o terapeuta e todos os membros da família; d) Deve ser um processo longo e contínuo durante o tratamento do paciente.

\section{Como são realizados}

O dia da reunião é marcado com antecedência, as famílias são convidadas a participar e a participação é espontânea. Participam, como psicoeducadores, médicos, enfermeiros, assistentes sociais e psicólogos. Os participantes comparecem no dia marcado, em local pré-estabelecido, no início da manhã, recebem a 
programação do dia. A programação é dividida nos períodos da manhã e da tarde no formato de aulas de vinte a trinta minutos. A divisão do dia está resumida no auadro a seguir:

\section{QUADRO 2 - DIVISÃO DO DIA DE WORKSHOP}

\begin{tabular}{|c|c|c|}
\hline PERIODO DA & E & $\mathrm{E}$ \\
\hline $\begin{array}{l}\text { - Do000000000000000000 } \\
\text { - P0000000000000 } \\
\text { - } 000000000000000000 \\
\text { 000000000000000000000 } \\
\text { - } 0000000000000000000000\end{array}$ &  & 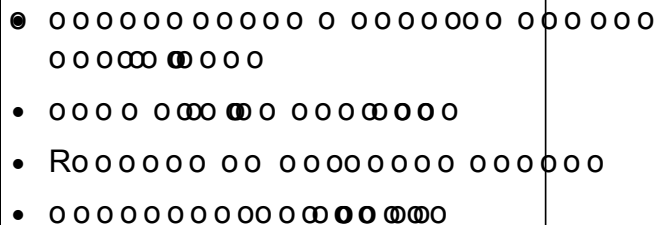 \\
\hline
\end{tabular}

As aulas do período da manhã fornecem um conteúdo de bases científicas da doença mental em questão, abordando aspectos etiológicos, diagnósticos, quadro clínico, tratamentos medicamentosos, psicoterápicos e psicossociais. Essas aulas transmitem aos familiares desde conceitos básicos até novos conhecimentos ainda em desenvolvimento. Este tipo de postura proporciona segurança e otimismo aos familiares que percebem que não estão submetidos a uma doença misteriosa ou desconhecida, diminuindo as falsas crenças e dando uma noção dos avanços que existem e que potencialmente podem ser desenvolvidos na área de saúde mental.

Ao final da manhã há uma pausa para descanso com duração de trinta minutos, e é servido um lanche ou almoço quando ocorre uma confraternização, na qual os participantes conversam entre si e com os psicoeducadores, podendo esclarecer dúvidas da aulas dadas e trocar experiências.

No período da tarde as aulas abordam aspectos humanos da doença mental: a relação da família com a doença mental, os sentimentos gerados pela doença, as reações adaptativas normais e anormais dos membros da família, como a família pode colaborar com o tratamento e com a reinserção social do paciente, melhores maneiras de controle de alteração de comportamento e detecção de sinais precoces de recaída.

Ao término das aulas os participantes são divididos em grupos pequenos, de no máximo oito pessoas, aos quais juntam-se um ou dois psicoeducadores e nesses grupos os familiares podem esclarecer dúvidas particulares, trocar experiências, fazer reclamações ou trazer novas idéias.

\section{CONCLUSÃO}

0 envolvimento familiar no tratamento dos pacientes que sofrem de doenças mentais está trazendo para a psiquiatria novos problemas, e com isto novas áreas de estudo. Como já foi descrito, relações familiares e doença mental vem sendo estudadas há pelo menos sessenta anos e a evolução do entendimento desta complexa interação traz importantes benefícios para os doentes mentais, suas famílias e profissionais de saúde mental. Atualmente as famílias e doentes mentais deveriam estar sendo vistos e apoiados por uma equipe multidisciplinar. Esta equipe permite uma atuação global que atenda as necessidades das famílias e pacientes. Estas estão sendo cada vez mais estudadas no sentido de permitir melhores meios de atendê-las e os resultado dos meios de atendimento também estão sendo cada vez mais documentados. Uma das necessidades primordiais destas pessoas (doentes mentais e família), é a de educação básica em saúde mental (Anderson et al, 1980). 0 desconhecimento e preconceito sobre doença mental são, ainda, os principais inimigos da participação familiar no tratamento de pacientes portadores de transtornos psiquiátricos.

0 desconhecimento sobre doença mental também colabora para a falta de investimentos materiais em todas as áreas de saúde e doença mental, desde tratamento primário até pesquisas e investimento em profissionais da área; uma vez que, famílias e pacientes estão alheios sobre a doença, sobre quais os benefícios que pesquisas e melhores meios de cuidados poderiam proporcionar.

A psicoeducação no formato de workshop proporciona um terreno fértil para o desenvolvimento de uma maior integração entre pacientes, famílias e profissionais de saúde mental na luta contra os prejuízos que a doença mental, quando tratada de maneira inadequada, traz não só para a unidade familiar mas para a sociedade 
como um todo.

ABSTRACT: The advances in psychiatric practice allowed patients to live in the community. The role of the family in the care of the mentally ill became more important. In the recent past the families were considered responsible for the cause of severe mental disorders, ideas that brought only problems for everybody involved, professionals families and patients.For the family to provide good care, basic education on mental disease is necessary. Psychoeducation brings benefits for the patients (decreasing relapses) and family (decreasing burden). The psychoeducation meetings are described as a way to provide important scientific information and exchange experiences.

KEY WORDS: Family health; Mental health; Psychology educational; Psychiatric nursing; F amily.

RESUMEN: Los avances obtenidos por la Psiquiatria en el tratamiento de personas con transtorno mental permitieron que, en los últimos 50 años, el número de internamientos y su duración disminuyan favoreciendo la vida próxima a la comunidad. Con esto el papel de la familia en el cuidado del enfermo mental se tornó más importante. La familia fué considerada en el pasado reciente causante o mediadora de la enfermedad mental. Esto generó grandes problemas para el tratamiento eficiente del paciente. Sin embargo, recientemente, el papel como responsable por el cuidado del enfermo mental viene siendo valorizado. Para esa actividad, la educación básica sobre la enfermedad mental es imprescindible. La psicoeducación familiar tiene beneficios para los pacientes (disminuye recaídas) y familiares (disminuye la sobrecarga). Las reuniones psicoeducativas, que aqui se describen, brindan informaciones científicas a las familias y permiten el intercambio de experiencias bajo la forma de clases y reuniones en pequeños grupos.

PALABRAS CLAVE: Salud de la familia; Salud mental; Psicologia educacional; Enfermeria psiquiatrica; Familia.

\section{REFERÊNCIAS}

1 KAVANAGH, D.J Recent developments in Expressed Emotion and Schizophenia. Br. J . Psychiatry, London, v.160, p.601-620, 1992.

2 ANDERSON, C. et al. Family treatment of adult schizophrenic: a psychoeducacional approach. Schizoph. Bull., R ockville, v.6, p.490-505, 1980.

3 ANDERSON, C. et al. Schizophrenia and the family. New York: Guilford, 1986.

4 BACHRACH, L.L.; CLARK, G.H. The first 30 years: a historical overview of community mental health. In: J .V. VACCAR 0 et al. Practicing psychiatry in the community. Washington: American Psychiatric Press, 1996, p. 3-27.

5 BATENSON, G. et al. EI medio intrafamiliar de los pacientes esquizofrenico: cisma marital y sesgo marital. In: BATENSON, G. et al. Doble vinculo y esquizofrenia. Buenos Aires, 1956. p.57-81.

6 BEARDSLEE, W.R. et al. Sustained change in parents receiving preventive interventions for families with depression. Am. J . Psychiatr., Washington, v.154, p.510-515, 1997.

7 BR OWN, G. et al. The post-hospital adjustment of chronic mental patients. Lancet, London, n.11, p. 685-694,1958.

8 BR OWN, G. et al. Influence of family life on the course of schizophrenia illness. Br. J . Prevent. Soc. Med. v.16, p.55-68, 1962.

9 BROWN, G.W.; RUTTER, M. The measurement of family activities and relationships: a methodological study. Human Relations, v.19, p.241-263, 1966.

10 CLARKIN, J .F. et al. Family and marital therapy. In: PAYKEL, E.S., Handbook of affective disorders. New York: Guilford, 1992, p.487-500.

11 DOMENICI, N.; GRIFFIN-FRANCELL C. The role of family education. J . Clin. Psychiatry, Menphis, v.54, n.3, supl, p.31-34, 1993.

12 FROMM-REICHMAN, F. Notes on the development of treatment of schizophrenics by psychoanalytical psychotherapy. Psychiatry, New York, n.11, p.263-273, 1948.

13 FROMM-REICHMAN, F. Contact whit relatives. In: Principles of intensive psychotherapy. Chicago: University of Chicago, 1960. p.214-224.

14 GABOR, I.K, P sichoeducational family intervention for depressed patients and their families. In: GABOR, I.K, Depression and 
families: impact and treatment, Washington: American Psychiatric, 1990. p.159-190.

15 HALFORD, W.K., Asessment of family interaction with a schizophrenic member. In: KAVANAGH, D.J . Schizophrenia: an overview and practical handbook. London: Chapman \& Hall, 1992. p.254-274.

16 HATFIELD, A.B. Psychological costs of schizophrenia to the family. Social Work, v.23, p.355-359, 1978.

17 HATFIELD, A.B. Coping and adaptation: a conceptual framework for understanding families. In: HATFIELD, A.B.; LEFLEY, H.P. Families of the mentally ill: coping an adaptation. New York: Guilford, 1987. p.60-84.

18 HOGARTY, G.E. et al. Family psychoeducation, social skills training, and maintenance chemotherapy in the aftercare treatment of schizophrenia: II. Two-years effects of a controlled study on relapse and adjustment .Arch. Gen. Psychiatry, Chicago, v.48, p.340-347, 1991.

19 HOGARTY, G.E. et al. Family psychoeducation, social skills training, and maintenance chemotherapy: I. One-year effects of a controlled study on relapse an expressed emotion. Arch. Gen. Psychiatry, Chicago, v.43, p.633-642, 1986.

20 J ACKSON, H.J . et al. Relationship between Expressed Emotion and family burden in psychotic disorders: an exploratory study. Acta Psychiatr. Scand., Copenhagen, v.82, p.243-249, 1990.

21 KASANIN, J . et al. Child relationship in schizophrenia. J ournal of Nervous and Mental Disease 121. Apud: MONTAGNA, P.L.K. (1982). Avaliação de emoções expressas em familiares de pacientes psiquiátricos. Revista da Associação Brasileira de Psiquiatria, n.4, p.75-80, 1934.

22 KEITNER, G.I. Psychoeducational family intervention for depressed patients and their families. In: KEITNER, G.I. Depression and families: impact and treatment. Washington: American Psychiatric, 1990. p.159-184.

23 KREISMAN, D.E.; J OY V.L. Family response to the mental illness of a relative: a review of the literature. Schizoph. Bull., Rockville, v.10, p.34-57, 1974.

24 KUIPERS, L.; BEBBINGTON P. Working in partnership: clinicians and carers in the management of longstanding mental illness. Oxford: Heinemann Medical Books, 1990.

25 LAING, R.S. Mystification, confusion, and conflict. In: BOSZOR MENY I-NAGY, I. et al. Intensive Family Therapy, New York: Harper, 1965. p.112-134.

$26 L E F F$, J e et al. The role of maintenance therapy and relatives expressed emotion in relapse of schizofhrenia: A two-year folow up. Br. J. Psychiatr., v.139, p.102-104, 1981.

27 LEVENE, J.E. et al. The perceived family burden scale: measurement and validation. Schizophr. Res., Amsterdam, v.22, p.151-157, 1996.

28 LIDZ, T. et al. The intrafamilial environment of the schizophrenic patient. Psychiatry. New York, v.20, p.329-342, 1957.

29 MCFARLANE, W.R. et al. From research to clinical practice: dissemination of New York state's family psychoeducation project. Hosp. Commun. Psychiatry, v.44, p.265-270, 1993.

30 MCFARLANE, W.R. et al. Multiple-family group and psychoeducation in the treatment of schizophrenia. Arch. Gen. Psychiatry, Chicago, v.52, p.679-687, 1996.

31 MIKLOWITZ, D.J . et al. Family factors and the course of bipolar affective disorder. Arch. Gen. Psychiatry, Chicago, v.45, p.225-231, 1988.

32 MINTZ, L.I. et al. Expressed emotion: a call for partnership among relatives, patients, and professionas. Schizoph. Bull., Rockville, v.13, p.227-235, 1987.

33 MUESER K.T.;GLYNN S.M. Behavioral family therapy for psichiatric disorders. Boston: Allyn and Bacon, 1995.

34 MUESER, K.T. et al. Coping whit negative symptoms of schizophrenia: patient and family perspectives. Schizoph. Bull., Rockville, v.23, p.329-339, 1997.

35 PENN, D.L.; MUESER K.T. Research update on the psychosocial treatment of schizophrenia. Am. J . Psychiatry, Washington, v.153, p.607-617, 1996.

36 SPANIOL, L. Coping strategies of family caregivers. In: HATFIELD, A.B et al. Families of the mentally ill: coping an adaptation. New York: Guilford, 1987. p.208-222.

37 WINEFIELD, H.R.; HARVEY, E.J. Needs of family caregivers in chronic schizophrenia. Schizophr. Bull., Washington, v.20, n.3, p.557-566, 1994.

38 WYNNE, L.C.; SINGER, M.T. Thought disorder an family relations of schizophrenics. Arch. Gen. Psychiatry, Chicago, v.9, p.191-198, 1963.

39 ZIPPLE, A.; SPANIL L. Current educational and supportive models of family intervention. In: HATFIELD, A. et al. Families of the mentally ill: coping an adaptation. New York: Guilford, 1987. p.261-277. 